\title{
Template-free Directional Growth of Single-Walled Carbon Nanotubes on a- and r-Plane Sapphire
}

\author{
Song Han, Xiaolei Liu, Chongwu Zhou* \\ Department of Electrical Engineering-Electrophysics, \\ University of Southern California, Los Angeles, California 90089
}

\section{Supporting Information}

Supporting Information Available:

1. Potential applications of aligned nanotube arrays;

2. AFM studies of step edges on the surfaces of sapphire substrates;

3. Surface atom layout of sapphire substrates;

4. Size distribution of catalyst particles. 


\section{Potential Applications of Aligned Nanotube Arrays}

There are many potential applications of aligned SWNT array samples. For example, highdensity individual SWNT transistors can be assembled by using top-down lithography techniques, and furthermore, integrated circuits and sensors of high performance can be fabricated with such aligned nanotube arrays, as shown in Figure S1.

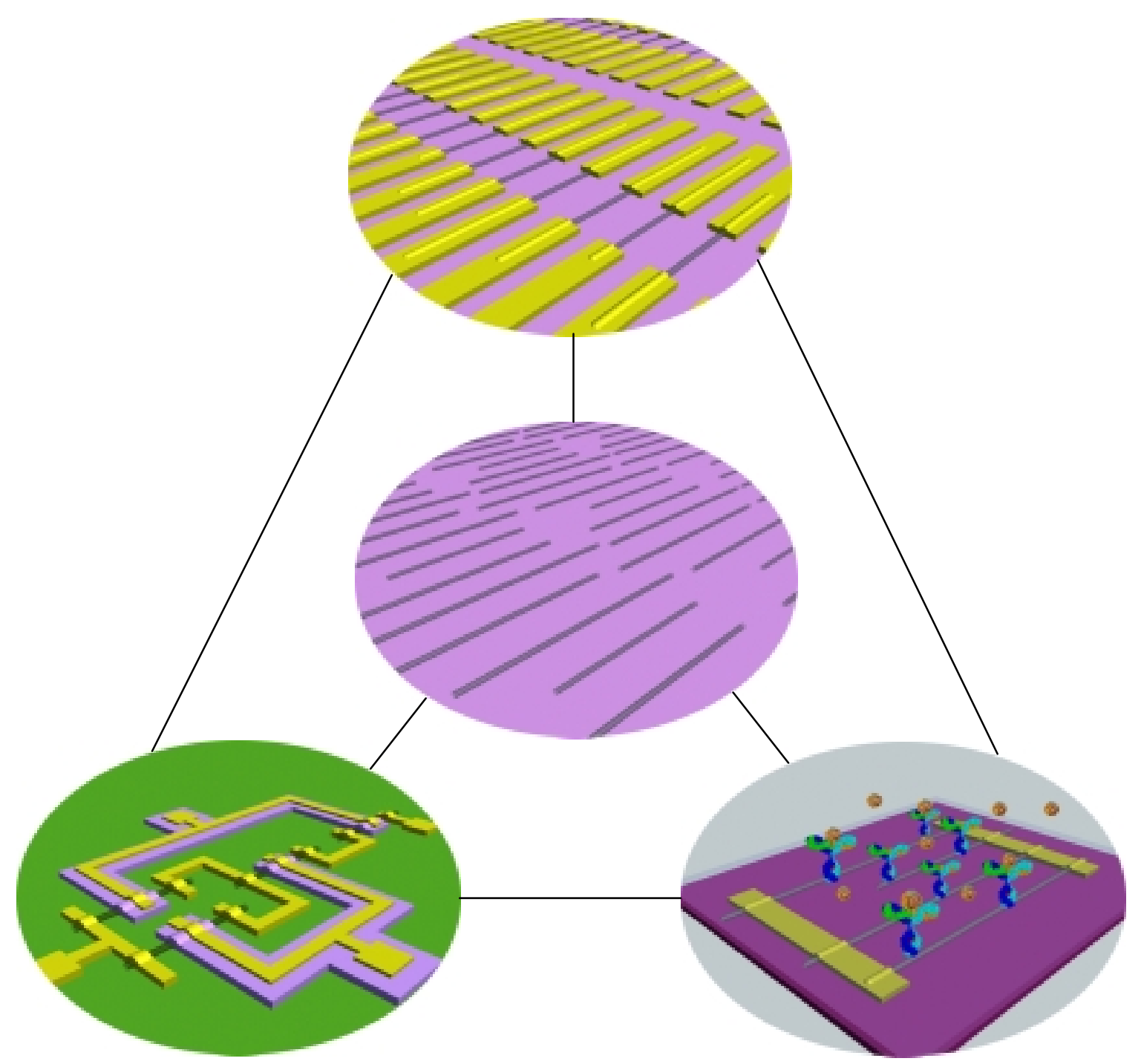

Figure S1. Potential applications of aligned SWNT arrays: high-density transistor arrays, integrated circuits and chemical / bio sensor arrays. 


\section{AFM Studies of Step Edges on the Surfaces of Sapphire Substrates}
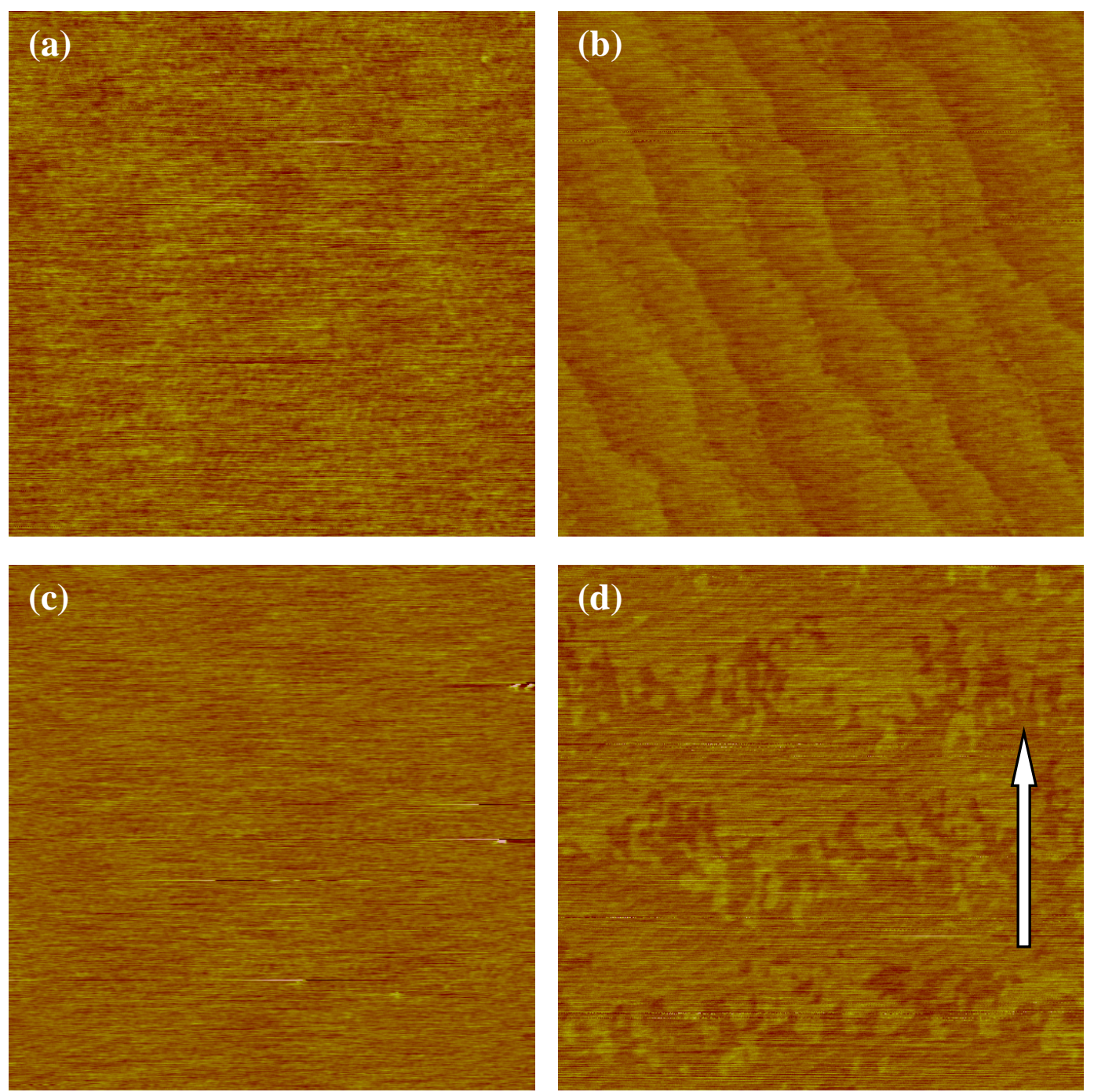

Figure S2. AFM images of sapphire substrates: (a) and (b) are the c-plane surfaces before and after annealing; (c) and (d) are the a-plane surfaces before and after the annealing. The size of all images is $1 \mu \mathrm{m} \times 1 \mu \mathrm{m}$. The arrow in (d) represents the nanotube growth direction.

We have carefully examined the role of step edges in the directional nanotube growth. Below we use results from c- and a-plane sapphire as the example, as the latter displayed highthroughput aligned nanotube growth, while the former displayed random orientations for the nanotubes. All the sapphire substrates were thoroughly cleaned by rinsing with acetone and oxygen plasma. The substrates were then inspected using AFM. In Figure S2a and c, both c- and 
a-plane substrates showed featureless flat surfaces, respectively. After the AFM inspection, the substrates were annealed at $1100{ }^{\circ} \mathrm{C}$ for 12 hours, a standard technique used to enhance the step edges. $^{7}$ The substrates were then examined using AFM again. As Figure S2b shows, parallel steps of about $0.5 \mathrm{~nm}$ in height can be found on annealed c-plane substrates. In contrast, for annealed a-plane sapphire substrates shown in Figure S2d, the step edges are rather irregular. Importantly, we found the growth direction of carbon nanotubes (indicated by the arrow in Figure S2d) on a-plane sapphire substrates is normal to the step edges. We have investigated samples of different batches and inspected multiple locations on each sample, and the results shown in Figure S2d are typical and reproducible. This convincingly demonstrates that the step edges do not play a role in the aligned nanotube growth we observed on a-plane sapphire substrates.

\section{Layout of atoms on sapphire substrates}

The origin of the orientation-controlled synthesis obtained with a-plane and r-plane sapphire deserves further study. We suggest the van der Waals interaction between the nanotubes and the substrates play an important role, similar to the orientation-controlled growth of nanotubes observed on silicon substrates. ${ }^{10}$ As shown in Figure S3a and b, oxygen atoms can be found to lie in the direction of aligned nanotube growth on a- and r-plane sapphire surfaces, respectively. In contrast, the atom density on m-plane sapphire surfaces (Figure S3c) is relatively low, while the atom layout on c-plane sapphire has six-fold symmetry. Both the atom density and the layout can affect the Lennard-Jones potential significantly. Detailed theoretical studies on the growth mechanism are currently undergoing and will be published elsewhere. 


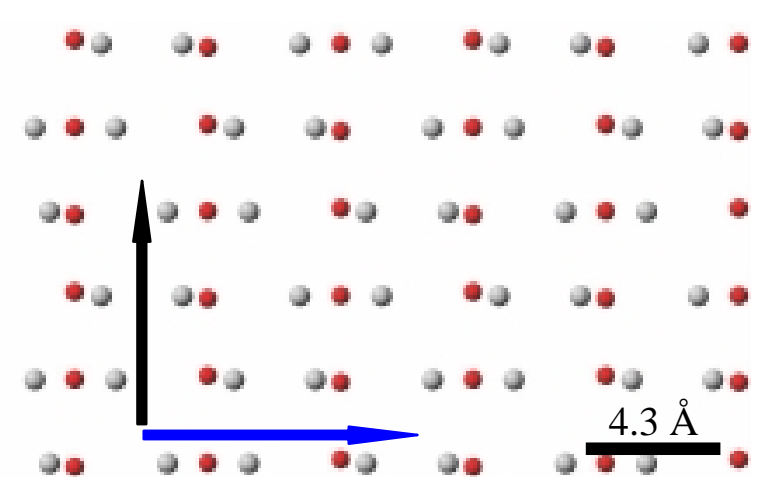

(a)

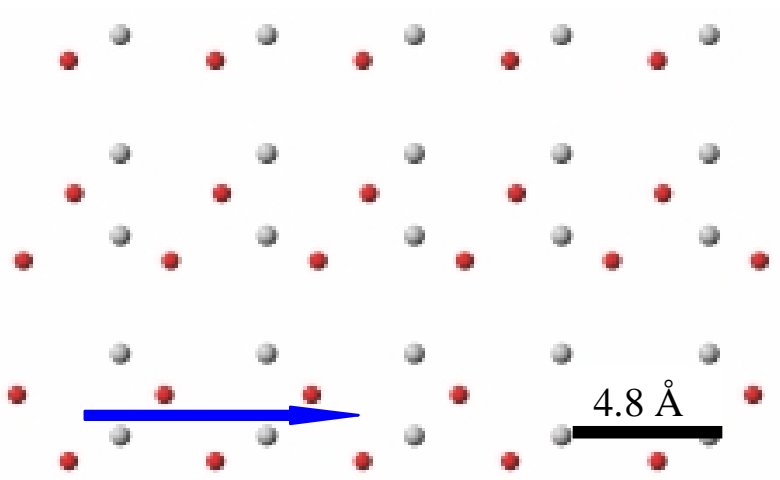

(c)

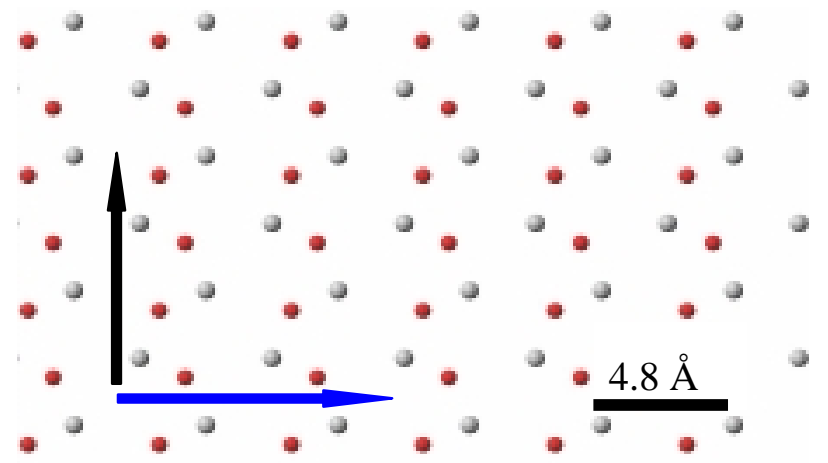

(b)

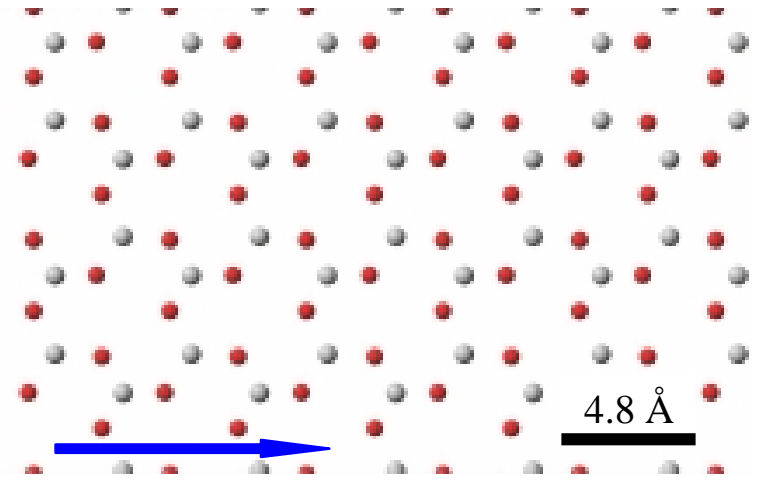

(d)

Figure S3. Layout of oxygen (red) and aluminum (light blue) atoms at the surfaces of (a) a-plane, (b) rplane, (c) m-plane and (d) c-plane sapphire substrates. The blue arrows represent (0001) direction in (a) and $(11 \overline{2} 0)$ direction in (b), (c) and (d). The black arrows in (a) and (b) show the aligned nanotube growth orientations experimentally observed with a-plane and r-plane sapphire, respectively. 


\section{Diameter Distribution of Catalyst Particles}

Diameter distribution of Ferritin clusters was studied using AFM. Figure S4 shows the diameter distribution of Ferritin catalyst right after calcinations at $700{ }^{\circ} \mathrm{C}$. The Gaussian fitting to the histogram gives diameter distribution as $2.88 \pm 0.83 \mathrm{~nm}$. The normalized diameter deviation is $\Delta D / D=0.29$, close to $\Delta D / D$ of 0.22 for the nanotubes (shown in Fig. $2 \mathrm{~d}$ ).

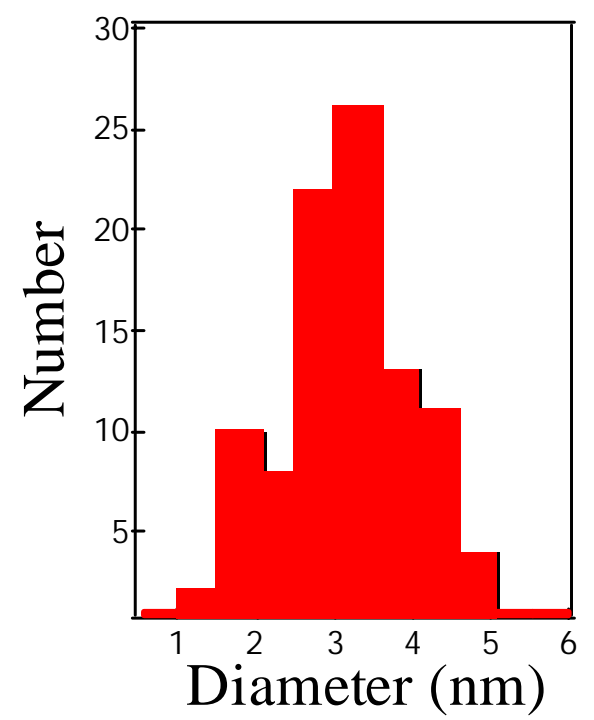

Figure S4. Histogram of the Ferritin cluster diameter distribution before the carbon nanotube growth. 